\title{
SEMIGROUP CLOSURES OF FINITE RANK SYMMETRIC INVERSE SEMIGROUPS
}

\author{
OLEG GUTIK, JIMMIE LAWSON, AND DUŠAN REPOVŠ
}

\begin{abstract}
We introduce the notion of semigroup with a tight ideal series and investigate their closures in semitopological semigroups, particularly inverse semigroups with continuous inversion. As a corollary we show that the symmetric inverse semigroup of finite transformations $\mathscr{I}_{\lambda}^{n}$ of the rank $\leqslant n$ is algebraically closed in the class of (semi)topological inverse semigroups with continuous inversion. We also derive related results about the nonexistence of (partial) compactifications of classes of semigroups that we consider.
\end{abstract}

\section{INTRODUCTION}

A partial one-to-one transformation on a set $X$ is a one-to-one function with domain and range subsets of $X$ (including the empty transformation with empty domain). There is a natural associative operation of composition on these transformations, $a b(x)=a(b(x))$ wherever defined, and the resulting semigroup is called the symmetric inverse semigroup $\mathscr{I}_{X}[9]$. The symmetric inverse semigroup was introduced by V. V. Wagner 31] and it plays a major role in the theory of semigroups. If the domain is finite and has cardinality $n$, which is then also the cardinality of the range, the transformation is said to be of rank $n$. For each $n \geq 0$, the members of $\mathscr{I}_{X}$ of rank less than or equal to $n$ form an ideal of $\mathscr{I}_{X}$, denoted $\mathscr{I}_{X}^{n}$. (Recall that a nonempty subset $I$ of a semigroup $S$ is an ideal if $S I \cup I S \subseteq I$.) If $X$ and $Y$ have the same cardinality, then these respective semigroups are isomorphic, and thus we may restrict our attention to a canonical one from that class, which we take to be the one arising by taking $X$ to be the cardinal $\lambda$. We thus label the corresponding semigroups as $\mathscr{I}_{\lambda}$ and $\mathscr{I}_{\lambda}^{n}$ respectively. The semigroups $\mathscr{I}_{\lambda}^{n}$ for $\lambda$ infinite form a motivating example for the considerations and developments of this paper.

Many topologists have studied topological properties of topological spaces of partial continuous maps $\mathscr{P} \mathscr{C}(X, Y)$ from a topological space $X$ into a topological space $Y$ with various topologies such as the Vietoris topology, generalized compact-open topology, graph topology, $\tau$-topology, and others (see [1, 7, 10, 12, 17, 18, 19, 20]). Since the set of all partial continuous self-transformations $\mathscr{P} \mathscr{C} \mathscr{T}(X)$ of the space $X$ with the operation composition is a semigroup, many semigroup theorists have considered the semigroup of continuous transformations (see surveys [21] and [14]), or the semigroup of partial homeomorphisms of an arbitrary topological space (see [2, 3, 4, 5, 13, 22, 27, 32]). Beŭda [6], Orlov [23, 24], and Subbiah [30] have considered semigroup and inverse semigroup topologies of semigroups of partial homeomorphisms of some classes of topological spaces. In this context the results of our paper yield some notable results about the topological behavior of the finite rank symmetric inverse semigroups sitting inside larger function space semigroups, or larger semigroups in general. For example, under reasonably general conditions, the inverse semigroup of partial finite bijections $\mathscr{I}_{\lambda}^{n}$ of rank $\leqslant n$ is a closed subsemigroup of a topological semigroup which contains $\mathscr{I}_{\lambda}^{n}$ as a subsemigroup.

Date: October 24, 2018.

2000 Mathematics Subject Classification. Primary 22A15, 20M20, 54H15. Secondary 20M12, 20M17, 20M18, $54 \mathrm{C} 25,54 \mathrm{D} 40$.

Key words and phrases. Topological semigroup, semitopological semigroup, topological inverse semigroup, symmetric inverse semigroup of finite transformations, algebraically closed semigroup, $\omega$-unstable set, semigroup with a tight ideal series. 
The class of semigroups that we consider in this paper is a general class of semigroups that is modeled on the semigroup $\mathscr{I}_{\lambda}^{n}$ and includes it as a special case. In Section 2 we take a closer look at this class including some categorical properties and a more general example than $\mathscr{I}_{\lambda}^{n}$.

A question of interest over the years has been to identify classes of semigroups that can be embedded in compact semigroups and classes that resist such embeddings. In Section 3 we consider this question in the context of the class of semigroups we are considering.

In this paper all topological spaces will be assumed to be Hausdorff. We shall follow the terminology of [8, 9, 11, 25, 26]. If $A$ is a subset of a topological space $X$, then we denote the closure of the set $A$ in $X$ by $\operatorname{cl}_{X}(A)$, or simply $\bar{A}$ if $X$ is obvious from context. By

$$
\left(\begin{array}{llll}
x_{1} & x_{2} & \cdots & x_{n} \\
y_{1} & y_{2} & \cdots & y_{n}
\end{array}\right)
$$

we denote a partial one-to-one transformation which maps $x_{1}$ onto $y_{1}, x_{2}$ onto $y_{2}, \ldots$, and $x_{n}$ onto $y_{n}$, and by 0 the empty transformation.

\section{Semigroup Closures}

Definition 1. A subset $D$ of a semigroup $S$ is said to be $\omega$-unstable if $D$ is infinite and for any $a \in D$ and infinite subset $B \subseteq D$, we have $a B \cup B a \nsubseteq D$.

The next lemma gives a basic example of $\omega$-unstable sets.

Lemma 2. For $\lambda$ infinite, $D:=\mathscr{I}_{\lambda}^{n} \backslash \mathscr{I}_{\lambda}^{n-1}$ is an $\omega$-unstable subset of $\mathscr{I}_{\lambda}^{n}$. Indeed for $a \in D$ and $B \subseteq D$ of cardinality at least $n !+1, a B \cup B a \nsubseteq D$.

Proof. In order for $a B$ to be contained in $D$, it must be the case that the range of each member of $B$ equals the domain of $a$ and in order for $B a$ to be contained in $D$, it must be the case the every member of $B$ have the same domain as the range $a$. Thus there are only $n$ ! possibilities for members of $B$.

Recall that a semigroup $S$ is a semitopological semigroup if it is equipped with a Hausdorff topology for which all left translation maps $\lambda_{s}$ and all right translation maps $\rho_{s}$ are continuous [26]. In this case we say equivalently that the multiplication is separately continuous. We come now to the crucial lemma for all that follows.

Lemma 3. Let $S$ be a semitopological semigroup, let $T$ be a subsemigroup, let $I$ be an ideal of $T$, and assume $D:=T \backslash I$ is $\omega$-unstable. If $s \in S$ is a limit point of $T$, then for each $t \in \bar{T}$, either $s t \in \bar{I}$ or $t s \in \bar{I}$.

Proof. We have $s \in \bar{T}=\bar{D} \cup \bar{I}$. If $s \in \bar{I}$, then by separate continuity of multiplication for each $t \in T$, st $\in \bar{I} t \subseteq \overline{I t} \subseteq \bar{I}$. Thus $s T \subseteq \bar{I}$, and by continuity of left translation by $s, s \bar{T} \subseteq \bar{I}$. Similarly $\bar{T} s \subseteq \overline{\bar{I}}$.

For the case that $s \in \bar{D}$, but $s \notin \bar{I}$, suppose for some $t \in T$ that $s t, t s \in W:=S \backslash \bar{I}$. Then $t \notin I$, for otherwise st, $t s \in \bar{I}$, and therefore $t \in D$. Using the continuity of left and right translation by $t$, we find an open set $U$ containing such that $U t \cup t U \subseteq W$. Since $s$ is a limit point of $T$ and $S$ is Hausdorff, the set $B:=T \cap(U \backslash \bar{I})$ is infinite. Since $B \subseteq T \backslash \bar{I} \subseteq D$ and $D$ is $\omega$-unstable, either $B t$ or $t B$ meets $I$. But $t B \cup B t \subseteq t U \cup U t \subseteq W$, and $W$ misses $I$, a contradiction. We conclude that for all $t \in T$, either $s t \in \bar{I}$ or $t s \in \bar{I}$. Thus the closed set $\left(\lambda_{s}\right)^{-1}(\bar{I}) \cup\left(\rho_{s}\right)^{-1}(\bar{I})$ contains $T$ and hence $\bar{T}$, which completes the proof.

Corollary 4. Let $S$ be a semitopological semigroup, let $T$ be a subsemigroup, let $I$ be an ideal of $T$, and assume $D:=T \backslash I$ is $\omega$-unstable. If $x, y \in \bar{T}$ and $x y x=x$, then either $x \in \bar{I}$ or $x \in T$ and $x$ is an isolated point of $\bar{T}$. 
Proof. Suppose that $x \notin \bar{I}$. If $x \notin T$, then $x$ must be a limit point of $T$. By Lemma 3 either $x y \in \bar{I}$ or $y x \in \bar{I}$. But then $x=(x y) x=x(y x) \in \bar{I}$, since $\bar{I}$ is an ideal of $\bar{T}$ by separate continuity. This contradicts our assumption that $x \notin \bar{I}$. Thus $x \in T \backslash \bar{I}$. If $x$ is a limit point of $T$, then Lemma 3 would again imply that $x=(x y) x=x(y x) \in \bar{I}$, a contradiction. Thus $x$ must be an isolated point of $T$ and hence also of $\bar{T}$.

Definition 5. An ideal series (see, for example, [9]) for a semigroup $S$ is a chain of ideals

$$
I_{0} \subseteq I_{1} \subseteq I_{2} \subseteq \ldots \subseteq I_{m}=S
$$

We call the ideal series tight if $I_{0}$ is a finite set and $D_{k}:=I_{k} \backslash I_{k-1}$ is an $\omega$-unstable subset for each $k=1, \ldots, m$.

Example 6. It follows from Lemma 2 that for an infinite cardinal $\lambda,\{\emptyset\} \subseteq \mathscr{I}_{\lambda}^{1} \subseteq \ldots \subseteq \mathscr{I}_{\lambda}^{m}$ is a tight ideal series for $S:=\mathscr{I}_{\lambda}^{m}$.

Recall that an element $x$ of a semigroup $S$ is regular if there exists $y \in S$ such that $x y x=x$ and that $S$ is regular if every element is regular [9]. If $x y x=x$, then it is straightforward to verify that $x^{\prime}=y x y$ is an inverse for $x$, i.e., $x x^{\prime} x=x$ and $x^{\prime} x x^{\prime}=x^{\prime}$. If $x$ belongs to an ideal $I$, then $x^{\prime}=y x y$ also belongs to the ideal, and it follows that an ideal of a regular semigroup is regular.

Proposition 7. Let $S$ be a semitopological regular semigroup that admits a tight ideal series $I_{0} \subseteq \ldots, \subseteq I_{m}=S$. Then each $I_{k}$ is closed in $S$ and each member of $S \backslash I_{m-1}$ is an isolated point of $S$.

Proof. We first prove by finite induction that $I_{k}$ is closed in $S$ for each $k$. First note that $I_{0}$ is closed since it is finite and $S$ is Hausdorff. Assume that $I_{k-1}$ is closed for some $k, 1 \leq k \leq m$. If $s \in \overline{I_{k}}$, then by regularity of the ideal $\overline{I_{k}}$ and Corollary $4 s \in \overline{I_{k-1}}=I_{k-1}$ or $s \in I_{k}$. In either case $s \in I_{k}$, so $I_{k}$ is closed. By induction $I_{k}$ is closed for all $k, 0 \leq k \leq m$. The last assertion now follows from Corollary 4.

By Example 6] and Proposition 7 we have the following

Corollary 8. Let $\lambda \geqslant \omega$ and let $n$ be any positive integer. If $\tau$ is a topology on $\mathscr{I}_{\lambda}^{n}$ such that $\left(\mathscr{I}_{\lambda}^{n}, \tau\right)$ is a semitopological semigroup, then every element $\alpha \in \mathscr{I}_{\lambda}^{n} \backslash \mathscr{I}_{\lambda}^{n-1}$ is an isolated point of the topological space $\left(\mathscr{I}_{\lambda}^{n}, \tau\right)$.

The next proposition establishes that semigroups with tight ideal series are restricted in regard to the type of semigroups in which they may be densely embedded.

Proposition 9. Let $S$ be a semitopological semigroup, let $T$ be a subsemigroup, and let $\bar{T}$ be its closure in $S$. If $T$ admits a tight ideal series, then any regular element of $\bar{T}$, in particular any idempotent, must already be in $T$.

Proof. Let $x$ be a regular element of $\bar{T}$, and let $I_{0} \subseteq \ldots \subseteq I_{m}=T$ be a tight ideal series for $T$. Let $k$ be the smallest index such that $x \in \overline{I_{k}}$. We claim $x \in I_{k}$. If $k=0$, then we are done since $I_{0}$ is finite, hence closed. So we assume $k \geq 1$. By hypothesis there exists $y \in \bar{T}$ such that $x y x=x$. Then $x^{\prime}:=y x y$ satisfies $x x^{\prime} x=x$ and $x^{\prime}=y x y \in \overline{I_{k}}$, since $\overline{I_{k}}$ is an ideal of $\bar{T}$. We apply Corollary 4 (with $T=I_{k}$ and $I=I_{k-1}$ ) and conclude that $x \in I_{k} \subseteq T$.

Recall that a semigroup is an inverse semigroup if it is a regular semigroup in which each element $x$ has a unique inverse $x^{\prime}\left[25\right.$. The semigroups $\mathscr{I}_{\lambda}$ and $\mathscr{I}_{\lambda}^{n}$ are inverse semigroups.

Proposition 10. Let $S$ be a semitopological inverse semigroup for which the inversion map $x \mapsto x^{\prime}$ is continuous. If $T$ is an inverse subsemigroup that admits a tight ideal series, then $T$ is closed in $S$. 
Proof. By separate continuity $\bar{T}$ is a subsemigroup. Since $T$ is closed under the inversion map and the inversion map is continuous, one readily sees that $\bar{T}$ is closed under inversion, i.e., $\bar{T}$ is an inverse, hence regular, subsemigroup. Proposition 9 then yields that $\bar{T} \subseteq T$, i.e., $T$ is closed.

Proposition 10 applies directly to the symmetric inverse semigroup $\mathscr{I}_{\lambda}^{n}$ for $\lambda$ infinite and $n$ a positive integer and yields the following corollary.

Corollary 11. Let $S$ be a semitopological inverse semigroup for which the inversion map $x \mapsto x^{\prime}$ is continuous. If (an isomorphic copy of) $\mathscr{I}_{\lambda}^{n}$ is a subsemigroup of $S$, then it is a closed subset of $S$.

Definition $12([15,28])$. Let $\mathfrak{S}$ be a class of topological semigroups. A topological semigroup $S \in \mathfrak{S}$ is called $H$-closed in the class $\mathfrak{S}$ if $S$ is a closed subsemigroup of any topological semigroup $T \in \mathfrak{S}$ which contains $S$ as a subsemigroup. If $\mathfrak{S}$ coincides with the class of all topological semigroups, then the semigroup $S$ is called $H$-closed.

We remark that in [28] the $H$-closed semigroups are called maximal.

Definition $13([15,29])$. Let $\mathfrak{S}$ be a class of topological semigroups. A semigroup $S$ is called algebraically closed in the class $\mathfrak{S}$ if for any topology $\tau$ on $S$ such that $(S, \tau) \in \mathfrak{S}$ we have that $(S, \tau)$ is an $H$-closed topological semigroup in the class $\mathfrak{S}$. If $\mathfrak{S}$ coincides with the class of all topological semigroups, then the semigroup $S$ is called algebraically closed.

We have immediately from Corollary 11 the following corollaries.

Corollary 14. For any infinite cardinal $\lambda$ and positive integer $n$, the semigroup $\mathscr{I}_{\lambda}^{n}$ is algebraically closed in the class of topological inverse semigroups (inverse semigroups that are topological semigroups with continuous inversion).

Corollary 15. Let $n$ be any positive integer and let $\tau$ be any inverse semigroup topology on $\mathscr{I}_{\lambda}^{n}$. Then $\left(\mathscr{I}_{\lambda}^{n}, \tau\right)$ is an $H$-closed topological inverse semigroup in the class of topological inverse semigroups.

The following example implies that for all $\lambda \geqslant \omega$, the semigroup $\mathscr{I}_{\lambda}^{k}$ with the discrete topology is not $H$-closed in the class of all locally compact topological semigroups, for any positive integer $k$.

Example 16. We fix any positive integer $k$. Let $a \notin \mathscr{I}_{\omega}^{k}$. Let $S=\mathscr{I}_{\omega}^{k} \cup\{a\}$. We put

$$
a \cdot a=a \cdot x=x \cdot a=0
$$

for all $x \in \mathscr{I}_{\omega}^{k}$.

We further enumerate the elements of the set $\omega$ by natural numbers. Let

$$
A_{m}=\left\{\left(\begin{array}{c}
2 l-1 \\
2 l
\end{array}\right) \mid l \geqslant m\right\}
$$

for each positive integer $m$. A topology $\tau$ on $S$ is now defined as follows:

1) all points of $\mathscr{I}_{\omega}^{k}$ are isolated in $S$; and

2) $\mathscr{B}(a)=\left\{U_{n}(a)=\{a\} \cup A_{n} \mid n=1,2,3, \ldots\right\}$ is the base of the topology $\tau$ at the point $a \in S$.

Then

a) for all $\left(\begin{array}{llll}x_{1} & x_{2} & \cdots & x_{i} \\ y_{1} & y_{2} & \cdots & y_{i}\end{array}\right) \in \mathscr{I}_{\omega}^{k}$ and $n \geqslant \max \left\{x_{1}, x_{2}, \ldots, x_{i}, y_{1}, y_{2}, \ldots, y_{i}\right\}$ we have

$$
\left(\begin{array}{llll}
x_{1} & x_{2} & \cdots & x_{i} \\
y_{1} & y_{2} & \cdots & y_{i}
\end{array}\right) \cdot U_{n}(a)=U_{n}(a) \cdot\left(\begin{array}{cccc}
x_{1} & x_{2} & \cdots & x_{i} \\
y_{1} & y_{2} & \cdots & y_{i}
\end{array}\right)=\{0\}
$$


b) $U_{n}(a) \cdot U_{n}(a)=U_{n}(a) \cdot\{0\}=\{0\} \cdot U_{n}(a)=\{0\}$ for any positive integer $n$; and

c) $U_{n}(a)$ is a compact subset of $S$ for each positive integer $n$.

Therefore $(S, \tau)$ is a locally compact topological semigroup. Obviously $\mathscr{I}_{\omega}^{k}$ is not a closed subset of $(S, \tau)$.

The following example shows that for all $\lambda \geqslant \omega$, the semigroup $\mathscr{I}_{\lambda}^{\infty}:=\bigcup_{n} \mathscr{I}_{\lambda}^{n}$ with the discrete topology is not $H$-closed in the class of all topological inverse semigroups.

Example 17. Let $\lambda \geqslant \omega$ and let $\tau_{d}$ be the discrete topology on the semigroup $\mathscr{I}_{\lambda}^{\infty}$.

For any $\varepsilon \in E\left(\mathscr{I}_{\lambda}^{\infty}\right)$ we define

$$
M(\varepsilon)=\left\{\chi \in \mathscr{I}_{\lambda}^{\infty} \mid \varepsilon \chi=\chi \varepsilon=\varepsilon\right\} .
$$

Let $S$ be the semigroup $\mathscr{I}_{\lambda}^{\infty}$ with the adjoined identity $\iota$. We now define a topology $\tau_{S}$ on the semigroup $S$ as follows:

(i) $\chi$ is an isolated point in $S$ for all $\chi \in \mathscr{I}_{\lambda}^{\infty}$; and

(ii) the family

$$
\mathscr{B}(\iota)=\left\{U_{\varepsilon}(\iota)=\{\iota\} \cup M(\varepsilon) \mid \varepsilon \in E\left(\mathscr{I}_{\lambda}^{\infty}\right)\right\}
$$

is the base of the topology $\tau_{S}$ at the point $\iota$.

The definition of the family $\mathscr{B}(\iota)$ implies that $\iota$ is not an isolated point of a topological space $\left(S, \tau_{S}\right)$ and the restriction of the topology $\tau_{S}$ on the set $\mathscr{I}_{\lambda}^{\infty}$ coincides with the topology $\tau_{d}$.

Obviously, this is sufficient to show that the semigroup operation on $\left(S, \tau_{S}\right)$ is continuous in the following cases:

(i) $\iota \iota=\iota$; and

(ii) $\iota \chi=\chi \iota=\chi$ for all $\chi \in \mathscr{I}_{\lambda}^{\infty}$.

In case $(i)$ we have

$$
U_{\varepsilon}(\iota) \cdot U_{\varepsilon}(\iota) \subseteq U_{\varepsilon}(\iota)
$$

In case $(i i)$ we denote

$$
\chi=\left(\begin{array}{llll}
x_{1} & x_{2} & \cdots & x_{n} \\
y_{1} & y_{2} & \cdots & y_{n}
\end{array}\right)
$$

Then we put

$$
K=\left\{x_{1}\right\} \cup\left\{x_{2}\right\} \cup \cdots \cup\left\{x_{n}\right\} \cup\left\{y_{1}\right\} \cup\left\{y_{2}\right\} \cup \cdots \cup\left\{y_{n}\right\} .
$$

Let be $K=\left\{a_{1}, a_{2}, \ldots, a_{k}\right\}$. Obviously $k \leqslant n$. We define

$$
\varepsilon=\left(\begin{array}{llll}
a_{1} & a_{2} & \cdots & a_{k} \\
a_{1} & a_{2} & \cdots & a_{k}
\end{array}\right) .
$$

Then we have $\chi \varepsilon=\varepsilon \chi=\chi$ and hence

$$
U_{\varepsilon}(\iota) \cdot \chi=U_{\varepsilon}(\iota) \cdot \chi=\{\chi\} .
$$

Since $\left(U_{\varepsilon}(\iota)\right)^{-1}=U_{\varepsilon}(\iota)$, we have that $\left(S, \tau_{S}\right)$ is a topological inverse semigroup which contains $\mathscr{I}_{\lambda}^{\infty}$ as dense inverse subsemigroup.

\section{Semigroups With tight ideal SERIES}

We have seen in the previous section that semigroups admitting a tight ideal series have interesting closure properties in larger semigroups. In this section we take a brief closure look at this class of semigroups, primarily to see that such semigroups extend signficantly beyond the finite rank symmetric inverse semigroups.

Lemma 18. The class of semigroups admitting a tight ideal series is closed under finite products. 
Proof. It suffices to check for the case $n=2$. Let $S$ have a tight ideal series $I_{0} \subseteq \ldots \subseteq I_{m}=S$ and $T$ have a tight ideal series $J_{0} \subseteq \ldots \subseteq J_{n}=T$. Set $K_{i}=I_{i} \times J_{0}$ for $0 \leq i \leq m$ and $K_{i}=S \times J_{i-m}$ for $m<i \leq n+m$. Then any infinite $B \subseteq K_{i+1} \backslash K_{i}$ has an infinite projection into either $S$ for $i \leq m$ and into $T$ for $i>m$, and since multiplication is coordinatewise, it directly follows that $a B \cup B a$ meets $K_{i}$.

Lemma 19. Let $h: S \rightarrow T$ be a surjective semigroup homomorphism such that each point inverse $h^{-1}(t)$ is finite. If $S$ has a tight ideal series, then so does $T$.

Proof. It is easy to see that $h^{-1}\left(I_{0}\right) \subseteq \ldots \subseteq h^{-1}\left(I_{m}\right)=S$ is a tight ideal series for $S$ if $I_{0} \subseteq \ldots \subseteq I_{m}$ is one for $T$.

Example 20. Let $\left\{X_{i} \mid i \in I\right\}$ be a collection of finite, pairwise disjoint sets, and let $X=\bigcup_{i \in I} X_{i}$. We consider the semigroup $\mathscr{P} \mathscr{T}(X, I)$ of partial functions on $X$ defined in the following way. First choose a subset $J \subset I$ and let $\alpha: J \rightarrow K$ be a bijection to another subset of $I$. A function $f: \bigcup_{i \in J} X_{i} \rightarrow \bigcup_{i \in K}$ is by definition in $\mathscr{P} \mathscr{T}(X, I)$ if and only if $x \in X_{i}$ implies $f(x) \in X_{\alpha(i)}$. All such partial functions ranging over all such $\alpha$ and corresponding $f$ form the subsemigroup $\mathscr{P} \mathscr{T}(X, I)$ under composition, a subsemigroup of the semigroup of all partial functions on $X$. We define such a function to have rank $n$ if the cardinality of the range (and hence domain) of $\alpha$ has cardinality $n$ and $\mathscr{P} \mathscr{T}(X, I)^{n}$ to be all functions of rank less than or equal to $n$. We then have an ideal series for the semigroup $\mathscr{P} \mathscr{T}(X, I)^{n}$ defined by $I_{k}=\mathscr{P} \mathscr{T}(X, I)^{k}$ for $0 \leq k \leq n$.

There is a surjective homomorphism $h$ from $\mathscr{P} \mathscr{T}(X, I)^{n}$ to $\mathscr{I}_{I}^{n}$, which assigns to $f \in$ $\mathscr{P} \mathscr{T}(X, I)^{n}$ the corresponding $\alpha: J \rightarrow K$ between the index sets. One checks directly that this homomorphism has finite point inverses, and hence it follows from Lemma 19 that the ideal series $I_{k}=\mathscr{P} \mathscr{T}(X, I)^{k}$ for $0 \leq k \leq n$ is tight.

We remark that the semigroup $\mathscr{P} \mathscr{T}(X, I)^{n}$ is regular, but not an inverse semigroup. It is well known that the semigroup of all transformations $\mathscr{T}(X)$ is regular [9, and essentially the same proof yields the regularity of $\mathscr{P} \mathscr{T}(X, I)^{n}$. Thus the principal results of Section 1 may be applied to the semigroup $\mathscr{P} \mathscr{T}(X, I)^{n}$.

\section{Compact Embeddings}

Several authors have considered the problem of showing that various specific semigroups or classes of semigroups do or do not embed into compact semigroups. For example one can use the Swelling Lemma to show that the bicyclic semigroup does not admit an embedding into a compact topological semigroup [8]. Closer to our current investigations, it was shown by Gutik and Pavlyk in [16] that an infinite topological semigroup of $\lambda \times \lambda$-matrix units $B_{\lambda}$ does not embed into a compact topological semigroup, every non-zero element of $B_{\lambda}$ is an isolated point of $B_{\lambda}$, and $B_{\lambda}$ is algebraically closed in the class of topological inverse semigroups. (This is essentially a special case of results of this paper for $\mathscr{I}_{\lambda}^{1}$.) However, we add a new wrinkle to earlier investigations by showing that certain partially compact embeddings do not exist, more precisely that the closure of certain embedded $\mathcal{D}$-classes cannot be compact.

Recall the Green's relations on a semigroup $S$. Two elements are $\mathcal{L}$-equivalent if they generate the same principal left ideal, i.e., $s \mathcal{L} t$ if $\{s\} \cup S s=\{t\} \cup S t$, and $\mathcal{R}$ related if they generate the same principal right ideal. (In the case that $s$ is regular the principal left ideal reduces to $S s$ since $s=s s^{\prime} s \in s S$.) The join of the equivalence relations $\mathcal{L}$ and $\mathcal{R}$ is denoted $\mathcal{D}$. It is a standard semigroup result that $\mathcal{D}$ is alternatively given by the relational compositions $\mathcal{L} \circ \mathcal{R}=\mathcal{R} \circ \mathcal{L}$ [9, Section 2.1]. A $\mathcal{D}$-equivalence class $D$ is called a regular $\mathcal{D}$-class if it contains a regular element. This is the case if and only if each $\mathcal{L}$-class and each $\mathcal{R}$-class contained in $D$ contains at least one idempotent if and only if every element of $D$ is regular [9, Chapter 2.3]. Furthermore, each inverse of a member of $D$ is back in $D$ [9, Chapter 2.3]

We recall a useful fact about regular $\mathcal{D}$-classes. 
Lemma 21. Let $a, c \in D$, a regular $\mathcal{D}$-class in a semigroup $S$. Then there exist $s, t \in D$ such that $c=$ sat.

Proof. Since $\mathcal{D}=\mathcal{R} \circ \mathcal{L}$, we may pick $b \in D$ such that $a \mathcal{R} b$ and $b \mathcal{L} c$. Pick an idempotent $e$ in the $\mathcal{R}$-class of $a$ and $u \in S$ such that $a u=e$. Since $e S=a S=b S$, we have also $e a=a$ and $e b=b$. For $t=u b$, we have $a t=a u b=e b=b$. Furthermore, $t=u b \in S b$ and $b=a t \in S t$, so $t \mathcal{L} b$, and thus $t \in D$. In a similar fashion one finds $s \in D$ such that $c=s b$. Then $c=s b=s a t$ and $s, t \in D$.

The next theorem is our main one on the non-existence of compact embeddings of certain $\mathcal{D}$-classes.

Theorem 22. Let $S$ be a topological semigroup and let $T$ be a subsemigroup having a tight ideal series $I_{0} \subseteq \ldots \subseteq I_{m}$. If $D:=I_{k+1} \backslash I_{k}$ is a regular $\mathcal{D}$-class, then $\bar{D}=\mathrm{cl}_{S}(D)$ is not compact.

Proof. Suppose the contrary. Then the infinite set $D$ has a limit point $x$ in the compact set $\bar{D}$. Let $x_{\alpha}$ denote a net in $D \backslash\{x\}$ converging to to $x$. For each $\alpha$, pick an inverse $x_{\alpha}^{\prime}$, which must again be in $D$. By compactness of $\bar{D}$, some subnet of $x_{\alpha}^{\prime}$ (which we again label $x_{\alpha}^{\prime}$ ) must converge to some $y \in \bar{D}$. By continuity of multiplication $x_{\alpha}=x_{\alpha} x_{\alpha}^{\prime} x_{\alpha} \rightarrow x y x$, and by uniqueness of limits $x=x y x$. Thus $x$ is a regular element.

Fix some $a \in D$. By Lemma 22 for each $\alpha$, there exists $s_{\alpha}, t_{\alpha} \in D$ such that $s_{\alpha} x_{\alpha} t_{\alpha}=a$. Again passing to convergent subnets, we have $s_{\alpha} \rightarrow s \in \bar{D}, t_{\alpha} \rightarrow t \in \bar{D}$, and $a=s_{\alpha} x_{\alpha} t_{\alpha} \rightarrow s x t$. Therefore $a=s x t$. In a similar fashion, we can write $x_{\alpha}=u_{\alpha} a v_{\alpha}$ and conclude $x=u a v$ for $u, v \in \bar{D}$.

It follows from Corollary 4 that $x \in \overline{I_{k}}$. Let $j$ be the smallest index such that $x \in \overline{I_{j}}$. Then $j \neq 0$, for otherwise since $I_{0}$ is finite, hence closed, $x \in I_{0}$, and hence $a=s x t \in I_{0}$, contradicting the fact that $a \notin I_{k}$, which contains $I_{0}$. Thus $j \geq 1$. If $x \notin I_{j}$, then $x$ would be a limit point of $I_{j}$, and hence a limit point of $I_{j} \backslash I_{j-1}$, since $x \notin \bar{I}_{j-1}$. Again by Corollary 4 it would follow that $x \in \bar{I}_{j-1}$, a contradiction. We conclude that $x \in I_{j}$, and then that $a=s x t \in \overline{I_{j}}$, since $\overline{I_{j}}$ is an ideal of $\bar{T}$. Applying Corollary 4 to the regular element $a$, we conclude either that $a \in I_{j}$, an impossibility since $I_{j} \subseteq I_{k}$, or $a \in \bar{I}_{j-1}$. The latter would imply $x=u a v \in \bar{I}_{j-1}$, which we have just seen is not the case. Thus we have reached a contradiction to our assumption that $\bar{D}$ is compact.

Since it is well-known and direct to verify that the sets $D=\mathscr{I}_{\lambda}^{k} \backslash \mathscr{I}_{\lambda}^{k-1}$ are $\mathcal{D}$-classes for $1 \leq k \leq n$ in the semigroup $\mathscr{I}_{\lambda}^{n}$, we have the following corollary, which generalizes the previously mentioned result of Gutik and Pavlyk in [16].

Corollary 23. For an infinite cardinal $\lambda$ and positive integer $n$, if $\mathscr{I}_{\lambda}^{n}$ is a subsemigroup of a topological semigroup $S$, it cannot be the case that that $\mathrm{cl}_{S}\left(\mathscr{I}_{\lambda}^{k} \backslash \mathscr{I}_{\lambda}^{k-1}\right)$ is compact for $1 \leq k \leq n$.

We close with a theorem on $\mathscr{I}_{\lambda}^{\infty}$.

Theorem 24. For any infinite cardinal $\lambda$ there exists no topology $\tau$ on $\mathscr{I}_{\lambda}^{\infty}$ such that $\left(\mathscr{I}_{\lambda}^{\infty}, \tau\right)$ is a compact semitopological semigroup.

Proof. Suppose to the contrary, that there exists a topology $\tau$ on $\mathscr{I}_{\lambda}^{\infty}$ such that $\left(\mathscr{I}_{\lambda}^{\infty}, \tau\right)$ is a compact semitopological semigroup.

The definition of the semigroup $\mathscr{I}_{\lambda}^{\infty}$ implies that for any idempotent $\varepsilon \in \mathscr{I}_{\lambda}^{\infty}$ there exists an idempotent $\phi \in \mathscr{I}_{\lambda}^{\infty}$ such that $\varepsilon \phi=\phi \varepsilon=\varepsilon$ and $\phi \neq \varepsilon$, i. e. $\varepsilon<\phi$. Therefore there exists a subsets of idempotents $A=\left\{\varepsilon_{1}, \varepsilon_{2}, \ldots, \varepsilon_{n}, \ldots\right\}$ in $\phi \in \mathscr{I}_{\lambda}^{\infty}$ such that

$$
0<\varepsilon_{1}<\varepsilon_{2}<\ldots<\varepsilon_{n}<\ldots
$$

Without loos of generality we can assume that $\varepsilon_{k} \in \mathscr{I}_{\lambda}^{k} \backslash \mathscr{I}_{\lambda}^{k-1}$, for any $k=2,3,4, \ldots$ and $\varepsilon_{1} \in \mathscr{I}_{\lambda}^{1} \backslash\{0\}$. Then Corollary 8 implies that the idempotent $\varepsilon_{k}$ has an open neighbourhood 
$U\left(\varepsilon_{k}\right)$ such that $U\left(\varepsilon_{k}\right) \cap \mathscr{I}_{\lambda}^{k}=\left\{\varepsilon_{k}\right\}$ for all $k=1,2,3, \ldots$ Since the translations in $\left(\mathscr{I}_{\lambda}^{n}, \tau\right)$ are continuous maps, the set

$$
U_{l}\left(\varepsilon_{k}\right)=\left\{\beta \in \mathscr{I}_{\lambda}^{\infty} \mid \beta \varepsilon_{k}=\varepsilon_{k}\right\}
$$

is clopen in the topological space $\left(\mathscr{I}_{\lambda}^{\infty}, \tau\right)$ for all $k=1,2,3, \ldots$. We define the family

$$
\mathscr{O}=\left\{O_{k} \mid k=1,2,3, \ldots\right\}
$$

as follows:

(i) $O_{1}=\mathscr{I}_{\lambda}^{\infty} \backslash U_{l}\left(\varepsilon_{1}\right)$; and

(ii) $O_{k}=U_{l}\left(\varepsilon_{k-1}\right) \backslash U_{l}\left(\varepsilon_{k}\right)$ for all $k=2,3,4, \ldots$

Obviously, the family $\mathscr{O}$ is a clopen cover of the topological space $\left(\mathscr{I}_{\lambda}^{\infty}, \tau\right)$, which does not contain a finite subcover, a contradiction.

\section{ACKNOWLEDGEMENTS}

This research was supported by SRA grants P1-0292-0101-04, J1-9643-0101 and BI-RU/0809-002.

\section{REFERENCES}

[1] A. Abd-Allah and R. Brown, A compact-open topology on partial maps with open domains, J. London Math. Soc. 21 (2) (1980), 480-486.

[2] B. B. Baird, Inverse semigroups of homeomorphisms between open subsets, J. Austral. Math. Soc. Ser. A 24:1 (1977), 92-102.

[3] B. B. Baird, Embedding inverse semigroups of homeomorphisms on closed subsets, Glasgow Math. J. 18:2 (1977), 199-207.

[4] B. B. Baird, Epimorphisms of inverse semigroups of homeomorphisms between closed subsets, Semigroup Forum 14:2 (1977), 161-166.

[5] B. B. Baird, Inverse semigroups of homeomorphisms are Hopfian, Canad. J. Math. 31:4 (1979), 800-807.

[6] A. A. Bel̆da, Continuous inverse semigroups of open partial homeomorphisms, Izv. Vyssh. Uchebn. Zaved. Mat. no. 1 (1980), 64-65 (in Russian).

[7] P. I. Booth and R. Brown, Spaces of partial maps, fibred mapping spaces and the compact-open topology, General Topology Appl. 8 (1978), 181-195.

[8] J. H. Carruth, J. A. Hildebrant and R. J. Koch, The Theory of Topological Semigroups, Vol. I, Marcel Dekker, Inc., New York and Basel, 1983; Vol. II, Marcel Dekker, Inc., New York and Basel, 1986.

[9] A. H. Clifford and G. B. Preston, The Algebraic Theory of Semigroups, Vol. I., Amer. Math. Soc. Surveys 7, Providence, R.I., 1961; Vol. II., Amer. Math. Soc. Surveys 7, Providence, R.I., 1967.

[10] A. Di Concilio nad S. Naimpally, Function space topologies on (partial) maps, Recent Progress in Function Spaces, D. Di Maio and Ľ. Holá (eds.), Quaderni di Mathematica, Vol. 3, Arace, 1998, 1—34.

[11] R. Engelking, General Topology, 2nd ed., Heldermann, Berlin, 1989.

[12] V. V. Filippov, Basic topological structures of the theory of ordinary differential equations, Topology in Nonlinear Analysis, Banach Center Publ. 35 (1996), 171-192.

[13] L. M. Gluskin, Semigroups of homeomorphisms, Dokl. Akad. Nauk UkrSSR. Ser. A no. 12 (1977), 10591061 (in Russian).

[14] L. M. Gluskin, B. M. Schein, L. B. Šneperman, and I. S. Yyaroker Addendum to a survey of semigroups of contionuous selfmaps, Semigroup Forum 14 (1977), 95-125.

[15] O. V. Gutik and K. P. Pavlyk, H-closed topological semigroups and topological Brandt $\lambda$-extensions, Math. Methods and Phys.-Mech. Fields 44:3 (2001), 20-28 (in Ukrainian).

[16] O. V. Gutik and K. P. Pavlyk, On topological semigroups of matrix units, Semigroup Forum 71:3 (2005), $389-400$.

[17] Ľ. Holá, Topologies on the space of partial maps, Recent Progress in Function Spaces, D. Di Maio and Ľ. Holá (eds.), Quaderni di Mathematica, Vol. 3, Arace, 1998, 157-186.

[18] Ľ. Holá, Complete metrizability of generalized compact-open topology, Topology Appl. 91:2 (1999), 159167.

[19] H. P. Künzi and L. B. Shapiro, On simulataneous extension of contionuous partial fuctions, Proc. Amer. Math. Soc. 125 (1997), 1853-1859.

[20] K. Kuratowski, Sur l'espace des fonctions partielles, Ann. Mat. Pura Appl. 40 (1955), 61—67. 
[21] K. D. Magill, Jr., A survey of semigroups of contionuous selfmaps, Semigroup Forum 11 (1975/1976), $189-282$.

[22] S. Mendes-Gonçalves and R. P. Sullivan, Maximal inverse subsemigroups of the symmetric inverse semigroup on a finite-dimensional vector space., Comm. Algebra 34:3 (2006), 1055-1069.

[23] S. D. Orlov, Topologization of the generalized group of open partial homeomorphisms of a locally compact Hausdorff space, Izv. Vyssh. Uchebn. Zaved. Matematika no.11(150) (1974), 61—68 (in Russian).

[24] S. D. Orlov, On the theory of generalized topological groups, Theory of Semigroups and its Applications, Sratov Univ. Press. no. 3 (1974), 80-85 (in Russian).

[25] M. Petrich, Inverse Semigroups, John Wiley \& Sons, New York, 1984.

[26] W. Ruppert, Compact Semitopological Semigroups: An Intrinsic Theory, Lecture Notes in Mathematics, Vol. 1079, Springer, Berlin, 1984.

[27] L. B. Šneperman, Semigroups of contionuous transformations and homeomorphisms of a simple arc, Dokl. Akad. Nauk SSSR 146 (1962), 1301-1304 (in Russian).

[28] J. W. Stepp, A note on maximal locally compact semigroups, Proc. Amer. Math. Soc. 20:1 (1969), 251-253.

[29] J. W. Stepp, Algebraic maximal semilattices, Pacific J. Math. 58:1 (1975), 243-248.

[30] S. Subbiah, The compact-open topology for semigroups of continuous self-maps, Semigroup Forum 35:1 (1987), 29-33.

[31] V. V. Wagner, Generalized groups, Dokl. Akad. Nauk SSSR 84 (1952), 1119-1122 (in Russian).

[32] I. S. Yaroker, Semigroups of homeomorphisms of certain topological spaces, Dokl. Akad. Nauk UkrSSR. Ser. A. no. 11 (1972), 1008-1010 (in Russian).

Department of Mechanics and Mathematics, Ivan Franko Lviv National University, UniverSYTETSKA 1, LVIV, 79000, UKRAINE

E-mail address: o_gutik@franko.lviv.ua

Department of Mathematics, Louisiana State University, Baton Rouge, LA 70803, USA

E-mail address: lawson@math.lsu.edu

Institute of Mathematics, Physics and Mechanics, and Faculty of Mathematics and Physics, University of Ljubljana, Jadranska 19, Ljubljana, 1000, Slovenia

E-mail address: dusan.repovs@guest.arnes.si 